\title{
Cibles et immunothérapies innovantes dans le traitement de l'allergie alimentaire
}

B. Bonnet ${ }^{1,2,3}$, P.A. Vinot ${ }^{1,2,3}$, B. Bellier ${ }^{1,2,3}$.

${ }^{1}$ Sorbonne Universités, UPMC Univ Paris 06, UMRS_959, I ${ }^{3}$, F-75013, Paris, France;

2 INSERM, UMRS_959, $1^{3}$, F-75013, Paris, France;

3 AP-HP, Groupe Hospitalier Pitié-Salpêtrière, Service de Biothérapies et Centre d'Investigation Clinique de Biothérapies, F-75013, Paris, France.

\section{Contact :}

Bertrand Bellier

Laboratoire d' Immunologie-Immunopathologie-Immunothérapie

INSERM UMRS959, Bat CERVI, Hopital Pitié-Salpêtrière

83 BD de l'Hôpital

75013 Paris

Tel. 0142177491

Mail. bertrand.bellier@upmc.fr

\section{Résumé}

Les pathologies allergiques constituent un problème de santé publique actuel majeur en raison d'une constante augmentation de leur prévalence dans les pays industrialisés. L'absence de traitement curatif efficace et le risque accru de choc anaphylactique suite à une nouvelle exposition à l'allergène font craindre une recrudescence des cas d'hospitalisation et des interventions médicales, en particulier pour les allergies alimentaires. Le développement de nouvelles thérapies permettant de prévenir et traiter les allergies alimentaires apparait donc comme une réelle nécessité.

La connaissance approfondie et grandissante des mécanismes physiopathologique des réactions allergiques permet d'offrir de nouvelles perspectives thérapeutiques. Les lymphocytes T régulateurs (Treg) constituent un parfait exemple. Un déficit quantitatif et/ou qualitatif de ces cellules a été observé chez les patients allergiques, pouvant expliquer en partie cette hypersensibilité aux allergènes. Ainsi, la stratégie consistant à recruter et activer in vivo des cellules aux fonctions suppressives offre une nouvelle piste thérapeutique. Dans cette optique, l'interleukine 2 (IL-2) est un candidat de choix puisque présentant des propriétés immunosuppressives lorsqu'elle est utilisée à faible dose. Les résultats cliniques obtenus récemment dans le cadre des maladies autoimmunes 
ainsi que le profil de sécurité associé motivent son évaluation chez l'homme pour d'autres dysfonctionnements immunitaires, y compris l'allergie alimentaire.

Au travers d'une revue de la littérature détaillant les principaux problèmes médicoépidémiologiques, les bases physiopathologiques de l'allergie alimentaire et les thérapies disponibles et à venir, nous détaillons l'intérêt de l'utilisation de faibles doses d'IL-2 en thérapie en exposant notamment les preuves de concepts et les différents essais cliniques réalisés à ce jour. 


\title{
Targets and innovative immunotherapies for the treatment of food allergy
}

\begin{abstract}
Allergic diseases are a major public health problem today due to a constant increase in their prevalence in industrialized countries. The absence of effective curative treatment and increased risk of anaphylactic shock following a new allergen exposure do fear a resurgence of hospitalizations and medical interventions, especially for food allergies. The development of new therapies to prevent and treat food allergies thus appears as a real need.

The extensive and growing knowledge of the pathophysiological mechanisms of allergic reactions can offer new therapeutic perspectives. Regulatory T cells (Tregs) are a perfect example. A quantitative deficit and / or quality of these cells was observed in allergic patients, which may partly explain the allergen hypersensitivity. Thus, the strategy to recruit and activate Tregs in vivo offers a new therapeutic path. In this context, interleukin 2 (IL-2) is a promising candidate as having immunosuppressive properties when used in low doses. The clinical results obtained recently in the context of autoimmune diseases and the associated safety profile motivates its evaluation in humans for other immune dysfunctions, including food allergy.

Through a literature review detailing the pathophysiological bases of food allergy, the available and future therapies, we show the interest of the use of low-dose IL-2 in therapy in particular outlining the evidence concepts and different clinical trials to date.
\end{abstract}




\section{Cibles et immunothérapies innovantes dans le traitement de l'allergie alimentaire}

\section{Table des matières}

Epidémiologie, aspects économiques et traitement actuels de l'allergie alimentaire

A. Epidémiologie. 1

B. Aspect économique 2

C. Traitements actuels de l'allergie 2

Nouvelles thérapies de l'allergie alimentaire 3

A. Physiopathologie et cibles thérapeutiques potentielles 3

B. Avancées thérapeutiques de l'allergie alimentaire. 6 Bibliographie 15

\section{Epidémiologie, aspects économiques et traitement actuels de l'allergie alimentaire}

L'allergie, aussi appelée hypersensibilité, est une réaction clinique observée lors d'une réponse immunitaire anormale contre un agent de provocation non pathogène appelé allergène. L'allergie alimentaire, manifestation clinique contre un allergène alimentaire (trophallergène), constitue un enjeu de santé publique majeur en raison d'une prévalence en constante augmentation comme les autres pathologies allergiques, mais également de la gravité éventuelle des signes cliniques et d'un arsenal thérapeutique pauvre.

\section{A. Epidémiologie}

Les plus fortes prévalences d'allergies alimentaires sont observées au sein des pays industrialisés et concernent majoritairement les populations jeunes. Si l’allergie peut apparaître à tout âge, elle reste plus fréquente chez l'enfant avec une prévalence de 7,1 \% (1) à $8 \%$ (2) dont 2,4 \% souffrant d'allergies alimentaires multiples (1) et $3 \%$ présentant des réactions sévères (1). Parmi la population adulte des pays industrialisés, entre $3 \%$ et $6,6 \%(2,3)$ des personnes souffrent d'allergies alimentaires. Alors que les données sur la prévalence de ces allergies dépendent avant tout de la définition des cas, aujourd'hui, différents éléments d'appréciation montrent que le nombre de personnes atteintes d'allergies alimentaires est en augmentation (4). Le nombre de patients atteints d'allergie alimentaire est estimé entre 200 et 250 millions de personnes dans le monde (3) et le 
nombre de formes sévères nécessitant une hospitalisation aurait été multiplié par 5 dans certains pays (5). En Europe, les allergies alimentaires constituent la cause principale d'anaphylaxie chez les enfants de 0 à 14 ans. Plus particulièrement en France, les déclarations d'anaphylaxies alimentaires sévères ont connu un accroissement de 28\% entre 2001 et 2006 (6). Ces nombres laissent présager la crainte d'une forte augmentation sur les prochaines années de cas graves avec risque de décès suite à un choc anaphylactique et conduisant à une augmentation des dépenses de santé dans ce domaine.

\section{B. Aspect économique}

La mesure de l'impact économique des allergies alimentaires est complexe en raison d'un défaut d'études économico-sanitaires fiables en Europe. En plus des coûts directs liés à la prise en charge médicale des patients, les coûts indirects alourdissent significativement l'impact économique de cette pathologie, prenant en compte l'absentéisme au travail et le manque de productivité du patient ou de ses proches.

Aux Etats-Unis, le coût global des allergies (concernant 50 millions d'individus selon le " Centers for Disease Control and Prevention») est estimé autour de plusieurs milliards de dollars par an. Les allergies alimentaires, quant à elles, représentent une perte de 225 millions et de 115 millions de dollars en coûts directs et indirects respectivement (7). En Europe, 80 millions d'individus sont atteints d'une des manifestations cliniques de l'allergie. L'impact financier est estimé autour de 100 milliards d'euros par an. L'essor de nouvelles thérapies pourrait donc avoir un réel impact financier.

\section{Traitements actuels de l'allergie}

Outre les traitements d'appoints, il existe dans le cadre des allergies alimentaires deux grands types de traitement prescrits aujourd'hui : le traitement reposant sur le régime d'éviction et le traitement du choc anaphylactique. L'immunothérapie spécifique (ou désensibilisation spécifique), qui est un traitement fréquemment utilisé dans le cadre des allergies respiratoires, est actuellement restreinte à peu d'indications de l'allergie alimentaire et n'est pratiquée que par certains services spécialisés.

\section{Traitements symptomatiques}

Les divers traitements pharmacologiques existants ne permettent qu'une prise en charge symptomatique de l'allergie. Parmi ces traitements, on retrouve en premier les antihistaminiques qui peuvent être prescrits dans différentes manifestations cliniques de l'allergie alimentaire: prurit, urticaire, eczéma, etc. Des médicaments anti-inflammatoires, dont les corticoïdes, sont également disponibles mais le plus souvent réservés aux manifestations sévères.

Dans le cadre des chocs anaphylactiques, l'adrénaline (ou épinéphrine), une catécholamine vasopressive est préconisée sous forme de stylos auto-injectables. La plupart des patients souffrant 
d'allergies graves sont informés du risque d'un potentiel choc anaphylactique et de la nécessité d'avoir à disposition immédiate un stylo auto-injecteur d'adrénaline. II y a cependant beaucoup d'obstacles au succès de son utilisation, malgré des recommandations claires. Selon les études, entre $62 \%$ et $87 \%$ des patients ne l'utilisent pas correctement (8) en raison d'une incapacité à reconnaître les symptômes d'anaphylaxie ou d'un manque de compréhension de la façon d'utiliser le stylo. Les patients sont également souvent porteurs d'un stylo périmé en raison de la faible fréquence d'utilisation.

Devant le peu de traitement disponible, l'anxiété générée et le mésusage de l'adrénaline, il est préférable d'agir sur la prévention de l'allergie. A ce jour, l'éviction de l'allergène est la seule approche préventive massivement possible.

\section{Eviction stricte de l'allergène}

L'éviction de l'allergène consiste en la stricte élimination de la consommation du ou des allergènes responsables de l'allergie alimentaire. Elle semble de principe simple, mais nécessite en pratique, plusieurs étapes avec notamment l'identification parfaite du ou des allergènes impliqués, l'éducation des patients, mais également dans le cas d'un enfant l'éducation des parents et du personnel des établissements scolaires méconnaissant souvent l'existence et les risques du choc anaphylactique (9). Toutefois, la prise en charge de l'allergie alimentaire par la stricte éviction de l'allergène est loin d'être la meilleure option thérapeutique. Dans l'enfance, les besoins nutritionnels pour assurer une bonne croissance et une bonne santé limitent les indications de cette stratégie et l'application d'un régime d'éviction peut entraîner de sévères malnutritions (10-12). II est donc nécessaire de développer des alternatives thérapeutiques moins contraignantes et impactant de façon moindre la qualité de vie. Seule une meilleure connaissance de la physiopathologie allergique et des mécanismes immunologiques impliqués ouvrent de nouvelles perspectives thérapeutiques.

\section{Nouvelles thérapies de l'allergie alimentaire}

De nouvelles biothérapies, et plus spécifiquement des immunothérapies, sont aujourd'hui en cours de développement. Elles ont toutes pour but de contrôler les réponses immunitaires exacerbées dirigées contre l'allergène mais ciblent différents mécanismes associés à la physiopathologie des allergies.

\section{A. Physiopathologie et cibles thérapeutiques potentielles}


La réponse allergique se caractérise par une hyperpolarisation de la réponse cellulaire $T$ vers une réponse de type Th2 médiée par I'IL-5, I'IL-4 et I'IL-13. Ce mécanisme est associé à la production d'immunoglobuline (Ig) de type E (IgE) après activation des lymphocytes B (LyB) par l'allergène. Ces cytokines sont par ailleurs responsables d'une augmentation du nombre de cellules effectrices dans les tissus cibles et le sang (mastocytes, basophiles et éosinophiles). Ceci constitue la phase de sensibilisation qui a lieu généralement au niveau du tractus digestif (13).

Les signes cliniques de l'allergie s'expliquent par la réintroduction et la reconnaissance de l'allergène par les IgE spécifiques préalablement fixées sur les effecteurs (mastocytes, basophiles) puis au pontage de ces immunoglobulines induisant la dégranulation de ces cellules. Ce phénomène d'exocytose, rapide et brutal, conduit à la sécrétion des médiateurs préformés (histamine, sérotonine, tryptase...) et néoformés (leucotriènes, prostaglandines et chimiokines), responsables des signes cliniques de l'allergie.

En dehors de ces mécanismes communs aux autres allergies, plusieurs facteurs spécifiques de l'allergie alimentaire sont néanmoins décrits. On peut citer notamment l'implication probable de la vitamine D dans l'apparition de la pathologie de par son effet dans le maintien de l'intégrité de la barrière épithéliale au niveau intestinal (14). Cette relation a été confirmée par la corrélation observée entre une carence de cette vitamine et l'apparition d'allergies à l'arachide dans une population infantile australienne. De plus, les enfants carencés présentaient également une plus forte probabilité de développement d'allergies alimentaires multiples (15).

Le microbiote au niveau intestinal semble également un facteur déterminant dans le développement de l'allergie alimentaire. Des modifications de la flore commensale pourraient déclencher l'apparition de pathologies allergiques alors qu'à l'inverse un enrichissement en Clostridia jouerait un rôle dans l'absence de sensibilisation et dans la restauration d'une tolérance orale (16). Ces données sont confortées par l'observation d'une hausse des allergies alimentaires au lait chez les enfants traités par plusieurs antibiothérapies durant la petite enfance et pendant la grossesse (17).

A ce jour, plusieurs biothérapies tendent à exploiter les propriétés régulatrices du système immunitaires afin de prévenir et traiter les allergies alimentaires. Parmi les cellules aux propriétés immunosuppressives, les lymphocytes $T$ régulateurs (Treg) constituent une population cellulaire essentielle dans le maintien de la tolérance immunitaire aux antigènes du soi et le contrôle des maladies auto-immunes et allergiques. Les Treg sont une sous-population de lymphocytes T CD4+ exprimant le CD25 et le facteur de transcription Foxp3. II a été rapporté qu'un défaut de Treg est souvent associé à des manifestations auto-immunes ou allergiques (13). Pour preuve, l'allergie est I'une des manifestations cliniques retrouvées chez les patients atteints du syndrome IPEX, caractérisé 
par un défaut de Treg en lien avec une mutation dans le gène codant pour Foxp3 (18). Un défaut qualitatif et quantitatif de Treg a également été reporté chez les patients allergiques en particulier chez les enfants à haut risque d'allergie (basé sur les prédispositions familiales notamment l'atopie maternelle) comparé aux enfants à faible risque (19). L'importance des Treg s'illustre par ailleurs dans leur rôle pour la mise en place de la tolérance aux allergènes alimentaires et dans la résolution spontanée des allergies au lait de l'enfant $(20,21)$. Dans le cadre de protocoles d'immunothérapie spécifique, une induction et une activation des Treg en lien avec l'efficacité thérapeutique confirme leur rôle dans le contrôle de l'allergie (22-24). Dans les modèles murins d'allergie alimentaire, l'élimination des Treg entraîne une exacerbation des signes cliniques de l'allergie alimentaire, démontrant leur rôle indispensable dans la régulation de ces manifestations (25-27). De même, l'administration préalable de Treg à des souris sensibilisées puis rendues allergique à l'ovalbumine s'est montrée très efficace quant à la prévention des signes cliniques de l'allergie alimentaire constituant la première démonstration de l'utilisation des Treg comme outil thérapeutique dans l'allergie alimentaire (25).

Plusieurs mécanismes sont décrits dans la régulation des allergies par les Treg (Figure 1). En premier lieu, les Treg limitent la présentation de l'allergène aux lymphocytes Th2 spécifiques $(28,29)$ et aux lymphocytes B (30), contrôlant notamment l'accessibilité aux molécules de co-stimulation via la molécule CTLA-4 qu'ils expriment à leur surface (31). Les Treg entraînent aussi une déviation de la production des IgE spécifiques vers la production d'IgG4 décrites comme protectrices (32). Les Treg inhibent le recrutement des cellules effectrices au niveau du site inflammatoire (13) et diminuent la dégranulation des mastocytes (33) ou de l'expression de FcعRI (34).

Clinical symptoms
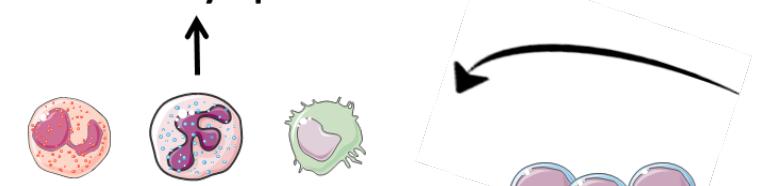

Eosinophils Basophils Mast cells

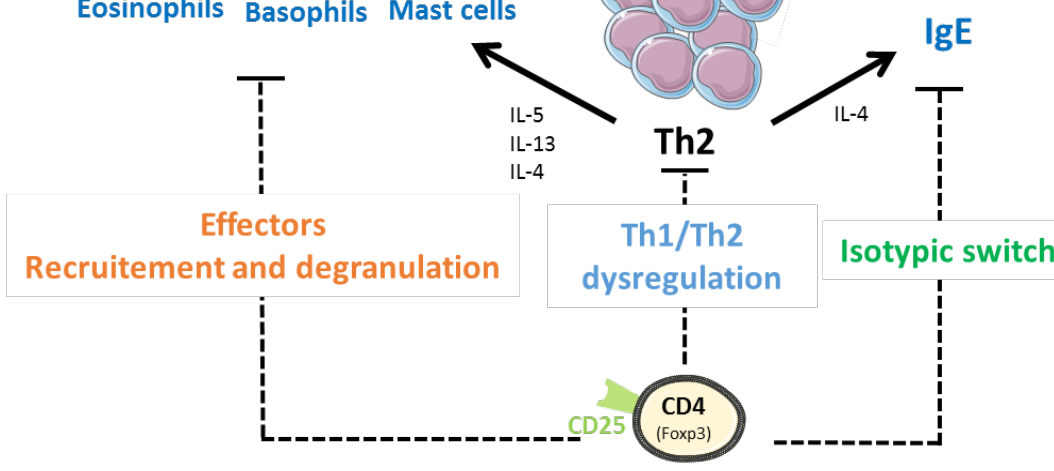

Treg
Figure 1 : Mécanismes d'action des lymphocytes $T$ régulateurs. Les lymphocytes T CD4+ Foxp3+ agissent à différents niveaux de la mise en place des réponses allergiques: de façon précoce agissant sur le compartiment Th2 associé à la sensibilisation ou de façon plus tardive agissant sur les cellules effectrices associées aux symptômes de l'allergie. Les différents mécanismes de suppression peuvent être également la cible de biothérapies. 
Ainsi, ces différents "checkpoints » de la physiopathologie de l'allergie constituent de nouvelles pistes de développement de biothérapies. Différentes approches sont proposées, comprenant les anticorps monoclonaux, la vaccination tolérogène, ou bien encore les immunothérapies immunomodulatrices.

\section{B. Avancées thérapeutiques de l'allergie alimentaire}

\section{Reconsidération de l'immunothérapie spécifique (ITS)}

L'ITS, communément appelée désensibilisation, constitue le pilier actuel du traitement de l'allergie sévère. Elle est la seule méthode actuelle permettant de prévenir et traiter durablement la maladie allergique notamment les allergies respiratoires mais les travaux actuels laissent à penser qu'elle pourra être proposée dans le futur pour l'allergie alimentaire.

L'ITS consiste à réduire la sensibilité de l'organisme à l'allergène en modulant progressivement la réponse immunitaire vis-à-vis de cet allergène notamment en rééquilibrant la balance Th1/Th2 via la diminution de la réponse Th2 et l'induction de la réponse T régulateurs (35). Pour cela, l'allergène est administré à dose croissante pour atteindre une dose efficace et permettre l'induction d'une tolérance spécifique. Plusieurs voies d'administration de l'allergène peuvent être utilisées dans le cadre de l'allergie alimentaire, présentant chacune un profil de tolérance et d'efficacité différent.

L'ITS par voie sous cutanée (SCIT) est sujette à controverse et n'est plus utilisée actuellement en raison d'un profil de sécurité insatisfaisant. De plus, seule une étude testant la SCIT dans le cadre d'une allergie aux poissons a été concluante avec l'utilisation de protéines recombinantes hypoallergéniques (36). Cette voie a donc logiquement été abandonnée et n'est plus proposée en routine clinique. Des considérations pratiques et logistiques ont également favorisé le développement d'alternatives moins contraignantes avec l'utilisation de la voie orale et sublinguale.

L'immunothérapie par voie orale (OIT) consiste en l'administration quotidienne à doses croissantes de l'allergène, sous forme de comprimé ou d'aliment natif, jusqu'à un maximum toléré. L'OIT est une technique très efficace augmentant significativement le seuil de la dose maximale tolérée lors d'un test de provocation oral chez $55 \%$ (37) des patients, à plus de $80 \%(38-40)$ selon les études et l'allergène. L'efficacité à long terme de l'OIT dépend fortement de l'allergène étudié et de la durée du traitement. Plus de $40 \%$ des patients réagissent de nouveau aux protéines de lait après 6 semaines d'OIT (40) alors que qu'après 5 ans de traitement $50 \%$ des patients sont toujours désensibilisés 1 mois après arrêt de l'OIT aux arachides, soulignant les limites actuelles de l'efficacité à long-terme de ces approches. Concernant la sécurité, 10 à 20 \% des patients enrôlés dans les essais cliniques d'OIT arrêtent le traitement en raison de réactions secondaires associées (41). Ces effets indésirables sont dans la plupart des réactions de grade I ou II $(42,43)$. De plus, la récente 
caractérisation des œsophagites à éosinophile réversibles après traitement a contribué à l'augmentation du nombre de réactions graves (44). Ces études démontrent clairement que l'OIT est efficace dans la désensibilisation, cependant la fréquence des réactions systémiques reste encore importante. De plus, un traitement prolongé, voire ininterrompu, semble nécessaire pour maintenir une efficacité à long terme satisfaisante.

L'immunothérapie par voie sublinguale (SLIT) présent l'avantage de cibler la muqueuse sublinguale qui constitue la première zone de contact avec le trophallergène, et est dite " pro-tolérogène " en raison de sa teneur en cellules dendritiques spécifiques capables d'induire des Treg. La SLIT consiste donc à administrer l'allergène en très faible quantité (de l'ordre du microgramme allant jusqu'au milligramme) à dose croissante sous la langue. Des concentrés d'extraits allergéniques en solution ou en tablettes sublinguales sont proposés (45). Les protocoles de désensibilisation sont très similaires à ceux de l'OIT (46). Les essais cliniques menés pour la plupart dans le cadre de l'allergie aux arachides ont été conduits durant 1 à 4 ans $(47,48)$ afin d'atteindre une dose maximale tolérée satisfaisante. En terme d'efficacité et comparativement à l'OIT, la SLIT présente une efficacité plus faible que I'OIT, néanmoins la sécurité d'emploi de la SLIT est supérieure à l'OIT avec un taux plus faible de réactions systémiques (49). Cependant, la SLIT n'a pas démontrée d'efficacité thérapeutique préventive tant sur le plan clinique que biologique (50).

La voie épicutanée est une voie récemment explorée qui a montré des résultats encourageants. En effet, les études suggérant que la peau constituait un site probable de sensibilisation aux allergènes alimentaires (51) ont conduit au développement d'une nouvelle voie d'administration de l'ITS. L'administration epicutanée de l'allergène, à l'aide de Patch, a clairement montré son potentiel tolérogénique dans des modèles animaux $(54,55)$. Chez l'Homme, différents essais cliniques sont en cours et les premiers résultats apparaissent prometteurs. Une étude pilote visant à démontrer la sécurité d'emploi de l'ITS par voie épicutanée (EPIT) lors d'un traitement de courte durée (90 jours) des allergies au lait a démontré une très bonne tolérance avec seulement comme effet secondaire des signes cutanés et gastro-intestinaux bénins (56). Un essai clinique de phase Ilb a également testé l'efficacité des patchs Viaskin Peanut ${ }^{\circledR}$ dans le cadre de l'allergie à la cacahuète, mettant en évidence une très bonne observance ainsi qu'une efficacité atteignant un taux de réponse de $40 \%$ chez les patients traités passant à $67 \%$ si l'analyse se concentre sur les enfants de 5 à 11 ans $(41,57)$. Ces premiers résultats soulignent l'efficacité de l'EPIT, qui est actuellement en étude de phase III, mais les taux d'efficacité rapportés restent similaires à ceux de la SLIT et inférieurs à ceux de l'OIT.

Globalement, I'ITS reste encore à être validée dans le cadre de l'allergie alimentaire et est de ce fait peu utilisée en routine clinique. L'efficacité relative, le manque de données économiques probantes 
lors des études coût-efficacité et l'hétérogénéité des protocoles d'immunothérapies constituent les limites actuelles, sans compter les risques associés à la réintroduction de l'allergène qui a conduit à délaisser cette thérapie durant ces dernières décennies.

\section{Anti-IgE}

Les thérapies visant à bloquer les IgE, acteur central dans les phénomènes allergiques, présentent un intérêt certain dans l'allergie alimentaire. Cependant une étude américaine évaluant l'omalizumab (anti-IgE) en monothérapie chez des patients atteints d'allergies à l'arachide fut interrompue suite aux réactions observées lors de challenges oraux (58). Malgré cet arrêt prématuré, des tendances de diminution d'IgE sériques chez les patients traités ont été observées. Aujourd'hui, l'omalizumab est souvent proposé en combinaison avec l'OIT afin de réduire les éventuelles réactions sévères liées à la réintroduction de l'allergène lors de la phase d'escalade de dose. Chez des patients présentant une allergie aux protéines de lait ou aux arachides et non-répondeurs à une première OIT, il a pu être montré que l'omalizumab administré comme adjuvant à une seconde OIT permettait de tolérer une dose d'allergène plus importante, de diminuer les effets indésirables de l'OIT ou encore de réduire le temps de désensibilisation. Cependant les résultats n'ont pas montré d'amélioration du taux de désensibilisation $(3,59,60)$. Enfin, une thérapie innovante visant à bloquer des IgE a été proposée par Pagovich \& al. Cette thérapie génique qui consiste en l'administration de vecteurs viraux autorisant l'expression d'anti-hlgE a démontré son efficacité dans un modèle préventif d'allergie à l'arachide chez des souris humanisées. Cette efficacité s'est traduite par la diminution des taux sériques d'IgE ainsi que la moindre sévérité des signes cliniques observés (61). Au final, I'utilisation d'anti-IgE semble être prometteuse même si des optimisations sont encore requises avant une utilisation à plus large échelle.

\section{Adjuvants pro-Th1}

De nouveaux protocoles d'ITS se développent associant l'administration de l'allergène avec des adjuvants pro-Th1, ciblant notamment les récepteurs Toll-like Receptor (TLR). Ces ITS adjuvantées ont pour but de favoriser le rééquilibre des réponses Th1/Th2 en renforçant les réponses Th1 (6264). Parallèlement à ces stratégies qui continuent d'inclure l'allergène, il a été testé l'administration simple d'antigène Mycobactérien, favorisant la réponse Th1, avec de l'ADN non-codant utilisé comme adjuvant pro-Th1 $(65,66)$. Ces immunothérapies qui s'affranchissent de la réintroduction de l'allergène, pratique considérée comme à risque chez les sujets allergiques, constituent un axe intéressant de recherche et pourrait bientôt intéresser les allergies alimentaires.

\section{Anti IL-5}


L'IL-5 fut longtemps considérée comme une cible intéressante dans la régulation des réponses allergiques. Impliquée dans le recrutement des populations responsables de l'inflammation locale allergique, sa neutralisation représente donc une piste prometteuse.

Les premiers essais utilisant les anticorps ciblant I'IL-5 (historiquement le mépolizumab) ont mis en avant son action bénéfique dans les crises d'asthme à éosinophiles réfractaire ainsi que dans la baisse du nombre d'éosinophiles sériques $(67,68)$. Ces effets thérapeutiques n'ont cependant pas été accompagnés d'amélioration de la fonction respiratoire et de plus n'ont pas été retrouvés chez des patients présentant de faibles taux d'éosinophiles (68). Par ailleurs, le mépolizumab a été étudié dans l'oesophagite à éosinophiles, maladie présentant des caractéristiques communes avec l'allergie alimentaire comme la présence d'IgE ou encore la disparition des symptômes après éviction de l'aliment incriminé. Une diminution significative des éosinophiles dans l'œsophage a été observée chez les patients traités par mépolizumab durant quelques jours (69). Néanmoins cette baisse ne s'est pas accompagnée d'une amélioration clinique ou d'une modification d'autres marqueurs biologiques. De ce fait, de nouvelles formulations visant à optimiser l'action de cet anticorps notamment par association à un autre anticorps ciblant I'IL-4 et/ou l'IL-13 (dupilumab) pourraient permettre de réévaluer son utilisation dans l'allergie alimentaire.

\section{Abatacept}

Les molécules de l'activation des lymphocytes $T$ et plus particulièrement les molécules de costimulation ont un rôle important dans l'initiation et l'entretien des réponses allergiques. Différentes stratégies ciblant ces molécules sont déployées, notamment le $\operatorname{CD} 28$, molécule de surface des lymphocytes T qui se lie aux CD80 et CD86 présents sur les cellules présentatrices d'antigènes et qui peut être bloqué par la molécule régulatrice CTLA-4. Les travaux de van Wijk et al. ont permis de montrer chez la souris l'importance de cette voie d'activation dans la génération d'une tolérance à la cacahuète (31). Ces recherches ont permis d'envisager l'utilisation d'inhibiteurs de cette voie dans le traitement des allergies. L'abatacept, molécule couplant le CTLA-4 à un domaine constant d'IgG humaine, constitue une approche thérapeutique intéressante de par son mode d'action, son profil d'utilisation et son efficacité dans d'autres modèles de maladies auto-immunes. En plus de réguler la polarisation Th2 et la production d'IgE, cette molécule peut agir directement sur l'inflammation. Malgré des résultats décevants en monothérapie dans l'asthme modéré chez l'homme (70), l'utilisation de l'abatacept dans I'allergie alimentaire en association avec d'autres thérapies reste intéressante.

\section{Microbiote \& Bactéries recombinantes}


L'étude extensive du microbiote ces dernières années et la découverte de ses propriétés immunorégulatrices ouvrent là encore de nouvelles pistes thérapeutiques pour l'allergie alimentaire. Alors que son rôle dans certaines pathologies auto-immunes est avéré, le microbiote reste aujourd'hui un candidat potentiel qui permet d'expliquer différentes situations de corrélation ou d'incidence exceptionnelle d'allergie telle le mode d'accouchement, l'âge de la diversification de la nourriture ou encore l'utilisation précoce et récurrente d'antibiotiques (71). L'étude de Cahenzli et al. a permis de démontrer le lien entre le microbiote et le niveau d'IgE chez l'Homme (72). Plusieurs hypothèses quant au mode d'action ont été proposées, notamment celle jouée par le microbiote comme barrière contre les allergènes, associée à une modulation de la plasticité et l'inflammation de l'épithélium intestinal (73). L'autre hypothèse émise est la capacité du microbiote à réguler la sécrétion d'IgE et à favoriser à l'inverse la sécrétion d'IgA, permettant un plus faible passage de l'allergène à travers la barrière mucosale (74). Au delà de la sphère intestinale, le microbiote présent en d'autres sites, comme la peau, pourrait également intervenir dans les phases de sensibilisation, y compris pour l'allergie alimentaire (71).

Plusieurs travaux ont exploité l'ensemble de ces propriétés régulatrices microbiennes afin d'induire une tolérance dans l'allergie. Différentes approches sont possibles combinant ou non l'administration mucosale et persistante d'un allergène, d'une molécule tolérogène ou l'utilisation des propriétés pro-tolérogènes de certaines souches bactériennes. Une des approches testées a permis d'évaluer l'impact d'une administration orale de Lactococcus Lactis recombinante sécrétant Der p2, un des peptides impliqués dans l'asthme allergique chez la souris. Les résultats ont permis de démontrer une efficacité dans la baisse des IgE spécifiques, ainsi que de l'inflammation locale pulmonaire (75). Une autre approche a consisté en I'utilisation de Lactococcus lactis capables de sécréter I'IL-10 au niveau local (76). Dans un modèle d'allergie alimentaire au lait chez la souris, l'administration orale de ces bactéries recombinantes a permis de faire diminuer les taux d'IgE et d'IgG1 sériques spécifiques de l'antigène (b-lactoglobulin). A l'inverse, des niveaux plus élevés d'IgA ont été retrouvés avec également une augmentation du taux d'IL-10 au niveau des plaques de Peyer. Ces résultats suggèrent la possibilité d'utiliser des agents pro-biotiques afin de prévenir voir de traiter les allergies alimentaires et d'envisager l'utilisation d'autres molécules tolérogènes. L'utilisation de bactéries recombinantes semble être une alternative de choix dans les allergies alimentaires tant par la possibilité de sécrétion de nombreuses molécules tolérogènes, d'allergènes que par l'étude de souches dotées de propriétés tolérogènes intrinsèques.

\section{Thérapie cellulaire ex vivo par Treg}

Le rôle central des Treg dans la régulation des réponses allergiques ayant été établi, de nouvelles thérapies spécifiques de ces cellules peuvent être envisagées. Jusqu'à récemment, ces approches de 
thérapie cellulaire par Treg reposaient sur l'expansion et la stimulation in vitro de Treg avant leur réadministration au patient. La preuve de concept a pu être apportée dans différents modèles précliniques chez l'animal $(25,77,78)$ mais ces stratégies restent confrontées aux problèmes de faisabilité en clinique humaine. L'obtention de population de Treg conservant leur fonction régulatrice après sélection et expansion ex vivo reste un point extrêmement critique. Alternativement aux stratégies de thérapie cellulaire ex vivo, une autre approche ciblant les Treg in vivo semble être bien plus prometteuse.

\section{IL-2 faible dose : une thérapie cellulaire in vivo spécifique des Treg}

II a pu être démontré que l'administration de faibles doses d'IL-2 a pour conséquence de recruter et d'activer spécifiquement la population suppressive de lymphocytes Treg in vivo.

\section{Rationnel et preuve de concept}

L'IL-2 est une cytokine de 15000 kDa produite par les cellules immunitaires (LT CD4, LT CD8, DC activées, NK et les NK exprimant le CD3 (NKT)) qui a été initialement décrite comme facteur de croissance des lymphocytes T. Elle agit en se fixant sur son récepteur composé de trois chaines polypeptidiques (IL-2R $\alpha$ (CD25), IL-2Rß (CD122) et IL-2Ry (CD132)) qui forment le récepteur de haute affinité à l'IL-2 (79). La fixation de l'IL-2 sur son récepteur induit alors une cascade de signalisation permettant la survie, la différenciation et l'expansion des lymphocytes T activés (79). Le dimère CD122 et CD132 peut également transduire le signal en l'absence de CD25, au prix d'une diminution d'affinité pour l'IL-2 (79). L'IL-2 a donc des effets variables selon la dose, s'expliquant par l'expression différentielle et spécifique du récepteur de haute affinité à la surface de certaines populations cellulaires. Le récepteur trimérique de haute affinité est constitutivement exprimé sur les Treg, tandis que les autres populations cellulaires sensibles à l'IL-2 acquièrent le CD25 qu'après activation (79). Les Treg sont donc plus sensibles à l'IL-2 que les autres populations cellulaires (Figure 2).

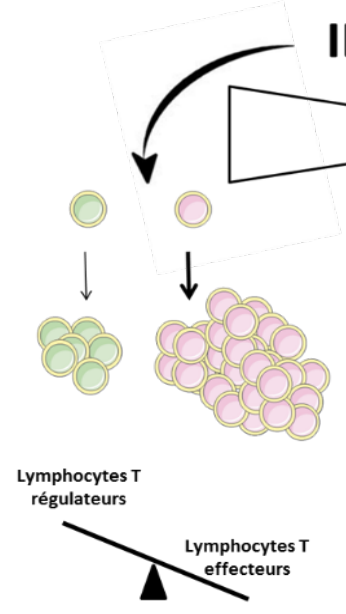

Immunostimulation
Figure 2. Les effets pléiotropiques de l'IL2 en fonction de la dose utilisée.

L'IL-2 utilisée à faible dose induit l'expansion préférentielle des Treg qui possèdent le récepteur de haute affinité pour I'IL-2. A l'inverse, I'IL-2 utilisée à forte dose entraîne également le recrutement et l'expansion des lymphocytes $T$ effecteurs. 
L'IL-2 à forte dose a d'abord été utilisée pour traiter les patients atteints de différents cancers (adénocarcinome rénal, mélanome métastatique sévère) afin de renforcer la réponse $T$ cytotoxique (Teff). Globalement, l'efficacité thérapeutique de l'IL-2 s'est avérée médiocre, notamment au vue des toxicités importantes qu'elle engendrait (80). De façon intéressante, des doses plus faibles d'IL-2 ont pu être proposées mais s'accompagnant d'une efficacité anti-tumorale limitée (réponse clinique faible, voire nulle) (81), qui peut aujourd'hui s'expliquer par une inversion de la balance Treg/Teff en faveur d'une augmentation des Treg aux faibles doses.

Ainsi, une faible dose d'IL-2 peut favoriser l'expansion des Treg tout en préservant les Teff. Les premiers travaux démontrant le potentiel immunosuppresseur de l'IL-2 faible dose ont été menés dans le cadre de l'auto-immunité. Grinberg-Bleyer et al. a montré que l'administration de faible dose d'IL-2 permettait de guérir les souris NOD (non obese diabetic) du diabète de type 1 s'expliquant par une augmentation des Treg et de leur capacité suppressive (82). L'IL-2 faible dose ou le complexe IL2/anti-IL2, favorisant l'expansion de Treg, ont ensuite été testés dans d'autres modèles expérimentaux de maladies auto-immunes (83-86) ou dans d'autres maladies inflammatoires (8789). Dans tous les cas, ce traitement s'est révélé très efficace et son efficacité a été associée à une activation in vivo spécifique des Treg et la sécrétion d'IL-10 (90). Le rôle anti-inflammatoire de l'IL-2 faible dose a également été montré dans le contrôle de maladies inflammatoires comme les lésions d'athérosclérose $(85,91)$, certaines dystrophies musculaires (88) ou encore certaines lésions pulmonaires aigues (92).

Chez l'homme, Saadoun et al. a montré que l'administration d'IL-2 à faible dose permettait d'augmenter spécifiquement les Treg chez des patients atteints de vascularite, s'accompagnant d'une amélioration clinique, constituant la première démonstration clinique de l'effet immunosuppresseur de I'IL-2. Suite à ces travaux, un essai clinique de phase I testant I'IL-2 faible dose dans le diabète de type 1 chez l'adulte a été mis en place. Les résultats préliminaires montrent un bon profil de tolérance et une augmentation des Treg de façon dose dépendante (93). Deux essais cliniques concernant le diabète chez l'enfant (NCT02411253 et NCT01862120, disponibles sur clinicaltrials.gov) et des patients atteints de maladies auto-immunes (étude de phase II concernant 11 pathologies auto-immunes et/ou inflammatoires ; NCT01988506) sont en cours.

En parallèle, d'autres groupes ont démontré l'efficacité clinique de l'administration de faibles doses d'IL-2 dans le traitement de la GVHD (94), du lupus (95) et des alopécies auto-immunes (96). Ces résultats prometteurs apportent la preuve que I'IL-2 faible dose constitue une bonne alternative à la thérapie cellulaire ex vivo.

Utilisation de faible dose d'IL-2 dans l'allergie. 
L'IL-2 possède des effets biologiques particulièrement intéressants, au regard des maladies allergiques intervenant sur plusieurs aspects de la physiopathologie sans l'inconvénient de la réadministration de l'allergène.

En effet, notre équipe a démontré que l'administration d'IL-2 faible dose permet l'expansion systémique (sang, rate) et locale (ganglion mésentérique, plaque de Peyer et intestin) des Treg chez la souris. De façon intéressante, cette expansion est sélective puisque les autres populations cellulaires exprimant le CD25 et potentiellement délétères dans l'allergie ne sont pas significativement impactées (Mastocytes, innate lymphoïd cells 2, Esoinophiles...) (26). D'autres études ont démontré que I'IL-2 bloque le développement de cellules pro-inflammatoire Th17 à partir des CD4 naîfs (97). De même, I'IL-2 bloque le développement des T Follicular helper (Tfh) (97), cellules impliquée dans la génération de la réponse anticorps/antigène spécifique. Enfin, il a été démontré que l'IL-2 active également les NK (79), cellules potentiellement impliquées dans la régulation des réponses $B$ et la production d'anticorps via l'inhibition des Tfh (98).

Les propriétés biologiques précédemment décrites, nous ont conduit à appliquer cette immunothérapie par IL-2 faible dose dans deux modèles murins d'allergie alimentaire (ovalbumine et cacahuète). L'efficacité préventive de l'IL-2 (administrée avant la phase de sensibilisation) a été attestée par une diminution de l'incidence et la sévérité des diarrhées et par une diminution de la chute de la température corporelle. Par ailleurs, l'effet thérapeutique de I'IL-2 faible dose est Tregdépendant dans la mesure où l'efficacité clinique de l'IL-2 chez les souris chez qui les Treg ont été éliminés est abrogée. IL-2 prévient le recrutement des effecteurs de l'allergie (éosinophiles, basophiles et mastocytes) et entraîne une diminution de la dégranulation des basophiles et des mastocytes mesurée respectivement par la diminution de l'expression membranaire du CD63 et la diminution du taux sérique de MCPT-1. Enfin, l'analyse des réponses immunitaires spécifiques de l'allergène montre que I'IL-2 permet le rééquilibre des réponses locales Th1/Th2 sans pour autant contrôler la sécrétion d'IgE spécifiques de l'Ovalbumine (26). L'efficacité curative de L'Il-2 faible dose (administrée après le déclenchement des signes cliniques) a été également démontrée dans ce modèle d'allergie à l'Ovalbumine (26). De façon remarquable, l'efficacité observée est complète et maintenue sur plusieurs mois après seulement deux cures d'IL-2 et ce aussi bien en préventif que thérapeutique.

L'administration de faible dose d'IL-2 a également été testée dans des modèles d'asthme allergique, démontrant l'efficacité préventive d'une cure de 5 jours d'IL-2 faible dose (50000UI) dans la prévention de l'hyperactivité bronchique chez des souris sensibilisée puis challengée par l'allergène (données non publiées). 


\section{IL-2 : adjuvant des ITS ?}

Dans la mesure où l'administration de faibles doses d'IL-2 faible induit l'expansion globale et transitoire des Tregs, et indépendamment de leur spécificité antigénique, se pose la question de la spécificité de la thérapie. Alors que dans le cadre des maladies auto-immunes, l'efficacité thérapeutique requiert la présence de Treg spécifiques des auto-antigènes (99), la présence et le rôle de Treg spécifiques des allergènes doivent être démontrés dans le cadre des allergies. Ainsi, I'administration simultanée d'IL-2 faible dose et de l'allergène pourrait être une stratégie optimale pour induire un plus grand nombre de Treg spécifiques et donc de gagner en efficacité thérapeutique. De façon intéressante, nous travaillons aujourd'hui sur la formulation de l'antigène afin de limiter les éventuelles réactions sévères suite à la réadministration de l'allergène et au contraire favoriser la tolérance.

\section{Conclusion}

Les évolutions épidémiologiques observées dans l'allergie alimentaire motivent aujourd'hui la recherche de nouveaux outils tant diagnostics que thérapeutiques rapidement disponibles pour les patients. En dehors de l'éviction stricte des allergènes pour les patients chez qui la cause a été identifiée, I'ITS reste à l'heure actuelle l'alternative thérapeutique la plus proposée. L'utilisation de nouvelles voies d'administration permet par ailleurs de moduler la balance efficacité-effets secondaires et de réduire les problématiques d'observance. Dans le domaine de la thérapeutique, les biothérapies représentent un domaine prometteur à la fois dans la prévention et dans la régulation des réponses allergiques observées. L'IL-2 à faible dose, par son recul d'utilisation et sa capacité à induire des réponses régulatrices, semble être un candidat intéressant. Les résultats préliminaires obtenus chez l'animal ainsi que la sécurité d'emploi de l'IL-2 faible dose à long terme (100) encouragent la définition de nouveaux schémas thérapeutiques et la conduite d'essais cliniques dans d'autres modèles d'animaux ainsi que chez l'homme. 


\section{Bibliographie}

1. Gupta RS, Springston EE, Warrier MR, Smith B, Kumar R, Pongracic J, et al. The prevalence, severity, and distribution of childhood food allergy in the United States. Pediatrics. 2011 Jul;128(1):e9-17.

2. Soller L, Ben-Shoshan M, Harrington DW, Fragapane J, Joseph L, St. Pierre Y, et al. Overall prevalence of self-reported food allergy in Canada. J Allergy Clin Immunol. 2012 Oct;130(4):986-8.

3. Umetsu DT, Rachid R, Schneider LC. Oral immunotherapy and anti-IgE antibody treatment for food allergy. World Allergy Organ J. 2015;8(1):20.

4. Sicherer SH, Sampson HA. Food allergy: Epidemiology, pathogenesis, diagnosis, and treatment. J Allergy Clin Immunol. 2014 Feb;133(2):291-307.e5.

5. Liew WK, Williamson E, Tang MLK. Anaphylaxis fatalities and admissions in Australia. J Allergy Clin Immunol. 2009 Feb;123(2):434-42.

6. Moneret-Vautrin D-A. Épidémiologie de l’allergie alimentaire. Rev Fr Allergol Immunol Clin. 2008 Apr;48(3):171-8.

7. Patel DA, Holdford DA, Edwards E, Carroll NV. Estimating the economic burden of food-induced allergic reactions and anaphylaxis in the United States. J Allergy Clin Immunol. 2011 Jul;128(1):110-5.e5.

8. O'Keefe AW, De Schryver S, Mill J, Mill C, Dery A, Ben-Shoshan M. Diagnosis and management of food allergies: new and emerging options: a systematic review. J Asthma Allergy. 2014;7:141-64.

9. Ercan H, Ozen A, Karatepe H, Berber M, Cengizlier R. Primary school teachers' knowledge about and attitudes toward anaphylaxis. Pediatr Allergy Immunol Off Publ Eur Soc Pediatr Allergy Immunol. 2012 Aug;23(5):428-32.

10. Groetch $M$, Nowak-Wegrzyn A. Practical approach to nutrition and dietary intervention in pediatric food allergy. Pediatr Allergy Immunol Off Publ Eur Soc Pediatr Allergy Immunol. 2013 May;24(3):212-21.

11. Flammarion S, Santos C, Guimber D, Jouannic L, Thumerelle C, Gottrand F, et al. Diet and nutritional status of children with food allergies. Pediatr Allergy Immunol Off Publ Eur Soc Pediatr Allergy Immunol. 2011 Mar;22(2):161-5.

12. Diamanti A, Pedicelli S, D'Argenio P, Panetta F, Alterio A, Torre G. latrogenic Kwashiorkor in three infants on a diet of rice beverages. Pediatr Allergy Immunol Off Publ Eur Soc Pediatr Allergy Immunol. 2011 Dec;22(8):878-9.

13. Palomares $\mathrm{O}$. The role of regulatory $\mathrm{T}$ cells in IgE-mediated food allergy. J Investig Allergol Clin Immunol. 2013;23(6):371-82; quiz 2 p preceding 382.

14. Kong J, Zhang Z, Musch MW, Ning G, Sun J, Hart J, et al. Novel role of the vitamin D receptor in maintaining the integrity of the intestinal mucosal barrier. AJP Gastrointest Liver Physiol. 2007 Oct 25;294(1):G208-16. 
15. Allen KJ, Koplin JJ, Ponsonby A-L, Gurrin LC, Wake M, Vuillermin P, et al. Vitamin D insufficiency is associated with challenge-proven food allergy in infants. J Allergy Clin Immunol. 2013 Apr;131(4):1109-16.e6.

16. Stefka AT, Feehley T, Tripathi P, Qiu J, McCoy K, Mazmanian SK, et al. Commensal bacteria protect against food allergen sensitization. Proc Natl Acad Sci U S A. 2014 Sep 9;111(36):1314550 .

17. Metsälä J, Lundqvist A, Virta LJ, Kaila M, Gissler M, Virtanen SM. Mother's and Offspring's Use of Antibiotics and Infant Allergy to Cow's Milk: Epidemiology. 2013 Mar;24(2):303-9.

18. Torgerson TR, Linane A, Moes N, Anover S, Mateo V, Rieux-Laucat F, et al. Severe food allergy as a variant of IPEX syndrome caused by a deletion in a noncoding region of the FOXP3 gene. Gastroenterology. 2007 May;132(5):1705-17.

19. Schaub B, Liu J, Höppler S, Haug S, Sattler C, Lluis A, et al. Impairment of T-regulatory cells in cord blood of atopic mothers. J Allergy Clin Immunol. 2008 Jun;121(6):1491-9, 1499.e1-13.

20. Karlsson MR, Rugtveit J, Brandtzaeg P. Allergen-responsive CD4+CD25+ regulatory $T$ cells in children who have outgrown cow's milk allergy. J Exp Med. 2004 Jun 21;199(12):1679-88.

21. Sletten GBG, Halvorsen R, Egaas E, Halstensen TS. Memory T cell proliferation in cow's milk allergy after $\mathrm{CD} 25$ + regulatory $T$ cell removal suggests a role for casein-specific cellular immunity in IgE-mediated but not in non-IgE-mediated cow's milk allergy. Int Arch Allergy Immunol. 2007;142(3):190-8.

22. Fujita $\mathrm{H}$, Soyka MB, Akdis M, Akdis CA. Mechanisms of allergen-specific immunotherapy. Clin Transl Allergy. 2012;2(1):2.

23. Smarr CB, Bryce PJ, Miller SD. Antigen-specific tolerance in immunotherapy of Th2-associated allergic diseases. Crit Rev Immunol. 2013;33(5):389-414.

24. Syed A, Garcia MA, Lyu S-C, Bucayu R, Kohli A, Ishida S, et al. Peanut oral immunotherapy results in increased antigen-induced regulatory $T$-cell function and hypomethylation of forkhead box protein 3 (FOXP3). J Allergy Clin Immunol. 2014 Feb;133(2):500-10.

25. Yamashita $\mathrm{H}$, Takahashi $\mathrm{K}$, Tanaka $\mathrm{H}$, Nagai $\mathrm{H}$, Inagaki $\mathrm{N}$. Overcoming food allergy through acquired tolerance conferred by transfer of Tregs in a murine model. Allergy. 2012 Feb;67(2):201-9.

26. Bonnet B, Vigneron J, Levacher B, Vazquez T, Pitoiset F, Brimaud F, et al. Low-Dose IL-2 Induces Regulatory T Cell-Mediated Control of Experimental Food Allergy. J Immunol Baltim Md 1950. 2016 Jun 3;

27. Kanjarawi R, Dercamp C, Etchart N, Adel-Patient K, Nicolas J-F, Dubois B, et al. Regulatory T cells control type I food allergy to Beta-lactoglobulin in mice. Int Arch Allergy Immunol. 2011;156(4):387-96.

28. Ling EM, Smith T, Nguyen XD, Pridgeon C, Dallman M, Arbery J, et al. Relation of CD4+CD25+ regulatory T-cell suppression of allergen-driven T-cell activation to atopic status and expression of allergic disease. Lancet. 2004 Feb 21;363(9409):608-15. 
29. Cottrez F, Hurst SD, Coffman RL, Groux H. T regulatory cells 1 inhibit a Th2-specific response in vivo. J Immunol Baltim Md 1950. 2000 Nov 1;165(9):4848-53.

30. Satoguina JS, Weyand E, Larbi J, Hoerauf A. T regulatory-1 cells induce IgG4 production by $B$ cells: role of IL-10. J Immunol Baltim Md 1950. 2005 Apr 15;174(8):4718-26.

31. Van Wijk F, Nierkens S, de Jong W, Wehrens EJM, Boon L, van Kooten P, et al. The CD28/CTLA4-B7 signaling pathway is involved in both allergic sensitization and tolerance induction to orally administered peanut proteins. J Immunol Baltim Md 1950. 2007 Jun 1;178(11):6894-900.

32. Meiler F, Klunker S, Zimmermann M, Akdis CA, Akdis M. Distinct regulation of IgE, IgG4 and IgA by T regulatory cells and toll-like receptors. Allergy. 2008 Nov;63(11):1455-63.

33. Gri G, Piconese S, Frossi B, Manfroi V, Merluzzi S, Tripodo C, et al. CD4+CD25+ regulatory T cells suppress mast cell degranulation and allergic responses through OX40-OX40L interaction. Immunity. 2008 Nov 14;29(5):771-81.

34. Kashyap M, Thornton AM, Norton SK, Barnstein B, Macey M, Brenzovich J, et al. Cutting edge: CD4 T cell-mast cell interactions alter IgE receptor expression and signaling. J Immunol Baltim Md 1950. 2008 Feb 15;180(4):2039-43.

35. Rolland JM, Gardner LM, O'Hehir RE. Allergen-related approaches to immunotherapy. Pharmacol Ther. 2009 Mar;121(3):273-84.

36. Zuidmeer-Jongejan L, Fernandez-Rivas M, Poulsen LK, Neubauer A, Asturias J, Blom L, et al. FAST: towards safe and effective subcutaneous immunotherapy of persistent life-threatening food allergies. Clin Transl Allergy. 2012;2(1):5.

37. Burks AW, Jones SM, Wood RA, Fleischer DM, Sicherer SH, Lindblad RW, et al. Oral immunotherapy for treatment of egg allergy in children. N Engl J Med. 2012 Jul 19;367(3):23343.

38. Caminiti L, Pajno GB, Crisafulli G, Chiera F, Collura M, Panasci G, et al. Oral Immunotherapy for Egg Allergy: A Double-Blind Placebo-Controlled Study, with Postdesensitization Follow-Up. J Allergy Clin Immunol Pract. 2015 Aug;3(4):532-9.

39. Varshney $\mathrm{P}$, Jones SM, Scurlock AM, Perry TT, Kemper A, Steele $\mathrm{P}$, et al. A randomized controlled study of peanut oral immunotherapy: clinical desensitization and modulation of the allergic response. J Allergy Clin Immunol. 2011 Mar;127(3):654-60.

40. Keet CA, Frischmeyer-Guerrerio PA, Thyagarajan A, Schroeder JT, Hamilton RG, Boden S, et al. The safety and efficacy of sublingual and oral immunotherapy for milk allergy. J Allergy Clin Immunol. 2012 Feb;129(2):448-55, 455.e1-5.

41. Chiang D, Berin MC. An Examination of Clinical and Immunologic Outcomes in Food Allergen Immunotherapy by Route of Administration. Curr Allergy Asthma Rep [Internet]. 2015 Jun [cited 2015 Jul 7];15(6). Available from: http://link.springer.com/10.1007/s11882-015-0536-y

42. Vazquez-Ortiz M, Alvaro M, Piquer M, Dominguez O, Machinena A, Martín-Mateos MA, et al. Baseline specific IgE levels are useful to predict safety of oral immunotherapy in egg-allergic children. Clin Exp Allergy J Br Soc Allergy Clin Immunol. 2014 Jan;44(1):130-41. 
43. Vázquez-Ortiz M, Alvaro-Lozano M, Alsina L, Garcia-Paba MB, Piquer-Gibert M, Giner-Muñoz $M T$, et al. Safety and predictors of adverse events during oral immunotherapy for milk allergy: severity of reaction at oral challenge, specific IgE and prick test. Clin Exp Allergy J Br Soc Allergy Clin Immunol. 2013 Jan;43(1):92-102.

44. Lucendo AJ, Arias A, Tenias JM. Relation between eosinophilic esophagitis and oral immunotherapy for food allergy: a systematic review with meta-analysis. Ann Allergy Asthma Immunol Off Publ Am Coll Allergy Asthma Immunol. 2014 Dec;113(6):624-9.

45. Senna GE, Calderon M, Milani M. Allergy immunotherapy tablet: Grazax ${ }^{\circledR}$ for the treatment of grass pollen allergy. Expert Rev Clin Immunol. 2011 Jan;7(1):21-7.

46. Kostadinova AI, Willemsen LEM, Knippels LMJ, Garssen J. Immunotherapy - risk/benefit in food allergy. Pediatr Allergy Immunol. 2013 Nov;24(7):633-44.

47. Marogna M, Spadolini I, Massolo A, Canonica GW, Passalacqua G. Long-lasting effects of sublingual immunotherapy according to its duration: a 15-year prospective study. J Allergy Clin Immunol. 2010 Nov;126(5):969-75.

48. Fleischer DM, Burks AW, Vickery BP, Scurlock AM, Wood RA, Jones SM, et al. Sublingual immunotherapy for peanut allergy: a randomized, double-blind, placebo-controlled multicenter trial. J Allergy Clin Immunol. 2013 Jan;131(1):119-27.e1-7.

49. Narisety SD, Frischmeyer-Guerrerio PA, Keet CA, Gorelik M, Schroeder J, Hamilton RG, et al. A randomized, double-blind, placebo-controlled pilot study of sublingual versus oral immunotherapy for the treatment of peanut allergy. J Allergy Clin Immunol. 2015 May;135(5):1275-82.e1-6.

50. Holt PG, Sly PD, Sampson HA, Robinson P, Loh R, Lowenstein H, et al. Prophylactic use of sublingual allergen immunotherapy in high-risk children: a pilot study. J Allergy Clin Immunol. 2013 Oct;132(4):991-3.e1.

51. Tordesillas L, Goswami R, Benedé S, Grishina G, Dunkin D, Järvinen KM, et al. Skin exposure promotes a Th2-dependent sensitization to peanut allergens. J Clin Invest. 2014 Nov;124(11):4965-75.

52. Brough HA, Simpson A, Makinson K, Hankinson J, Brown S, Douiri A, et al. Peanut allergy: effect of environmental peanut exposure in children with filaggrin loss-of-function mutations. J Allergy Clin Immunol. 2014 Oct;134(4):867-75.e1.

53. Fox AT, Sasieni $P$, du Toit $G$, Syed H, Lack G. Household peanut consumption as a risk factor for the development of peanut allergy. J Allergy Clin Immunol. 2009 Feb;123(2):417-23.

54. Dioszeghy V, Mondoulet L, Dhelft V, Ligouis M, Puteaux E, Benhamou P-H, et al. Epicutaneous immunotherapy results in rapid allergen uptake by dendritic cells through intact skin and downregulates the allergen-specific response in sensitized mice. J Immunol Baltim Md 1950. 2011 May 15;186(10):5629-37.

55. Dioszeghy V, Mondoulet L, Dhelft V, Ligouis M, Puteaux E, Dupont $\mathrm{C}$, et al. The regulatory $\mathrm{T}$ cells induction by epicutaneous immunotherapy is sustained and mediates long-term protection from eosinophilic disorders in peanut-sensitized mice. Clin Exp Allergy J Br Soc Allergy Clin Immunol. 2014 Jun;44(6):867-81. 
56. Dupont $\mathrm{C}$, Kalach $\mathrm{N}$, Soulaines $\mathrm{P}$, Legoué-Morillon S, Piloquet $\mathrm{H}$, Benhamou P-H. Cow's milk epicutaneous immunotherapy in children: a pilot trial of safety, acceptability, and impact on allergic reactivity. J Allergy Clin Immunol. 2010 May;125(5):1165-7.

57. Sampson HA, Agbotounou W, Thébault C, Charles R, Martin L, Yang WH, et al. Epicutaneous Immunotherapy (EPIT) Is Effective and Safe to Treat Peanut Allergy: A Multi-National DoubleBlind Placebo-Controlled Randomized Phase Ilb Trial. J Allergy Clin Immunol. 2015 Feb;135(2, Supplement):AB390.

58. Sampson HA, Leung DYM, Burks AW, Lack G, Bahna SL, Jones SM, et al. A phase II, randomized, double blind, parallel group, placebo controlled oral food challenge trial of Xolair (omalizumab) in peanut allergy. J Allergy Clin Immunol. 2011 May;127(5):1309-10.e1.

59. Yee CSK, Rachid R. The Heterogeneity of Oral Immunotherapy Clinical Trials: Implications and Future Directions. Curr Allergy Asthma Rep. 2016 Feb 27;16(4):1-19.

60. Wood RA, Kim JS, Lindblad R, Nadeau K, Henning AK, Dawson P, et al. A randomized, doubleblind, placebo-controlled study of omalizumab combined with oral immunotherapy for the treatment of cow's milk allergy. J Allergy Clin Immunol [Internet]. [cited 2015 Dec 14]; Available from: http://www.sciencedirect.com/science/article/pii/S0091674915014384

61. Pagovich OE, Wang B, Chiuchiolo MJ, Kaminsky SM, Sondhi D, Jose CL, et al. Anti-hlgE gene therapy of peanut-induced anaphylaxis in a humanized murine model of peanut allergy. $J$ Allergy Clin Immunol. 2016 Jun 23;

62. Wheeler AW, Marshall JS, Ulrich JT. A Th1-inducing adjuvant, MPL, enhances antibody profiles in experimental animals suggesting it has the potential to improve the efficacy of allergy vaccines. Int Arch Allergy Immunol. 2001 Oct;126(2):135-9.

63. Mothes N, Heinzkill M, Drachenberg KJ, Sperr WR, Krauth MT, Majlesi Y, et al. Allergen-specific immunotherapy with a monophosphoryl lipid A-adjuvanted vaccine: reduced seasonally boosted immunoglobulin E production and inhibition of basophil histamine release by therapyinduced blocking antibodies. Clin Exp Allergy J Br Soc Allergy Clin Immunol. 2003 Sep;33(9):1198-208.

64. Crivellaro M, Senna GE, Pappacoda A, Vanzelli R, Spacal B, Marchi G, et al. Safety of ultrashortterm sit with pollen allergoids adjuvanted by monophosphoryl lipid $\mathrm{A}$ : a prospective Italian survey. Eur Ann Allergy Clin Immunol. 2011 Mar;43(2):58-60.

65. Creticos PS, Schroeder JT, Hamilton RG, Balcer-Whaley SL, Khattignavong AP, Lindblad R, et al. Immunotherapy with a Ragweed-Toll-Like Receptor 9 Agonist Vaccine for Allergic Rhinitis. N Engl J Med. 2006 Oct 5;355(14):1445-55.

66. Fonseca DM, Wowk PF, Paula MO, Gembre AF, Baruffi MD, Fermino ML, et al. Requirement of MyD88 and Fas pathways for the efficacy of allergen-free immunotherapy. Allergy. 2015 Mar;70(3):275-84.

67. Haldar P, Brightling CE, Hargadon B, Gupta S, Monteiro W, Sousa A, et al. Mepolizumab and exacerbations of refractory eosinophilic asthma. N Engl J Med. 2009 Mar 5;360(10):973-84.

68. Nair P, Pizzichini MMM, Kjarsgaard M, Inman MD, Efthimiadis A, Pizzichini E, et al. Mepolizumab for prednisone-dependent asthma with sputum eosinophilia. N Engl J Med. 2009 Mar 5;360(10):985-93. 
69. Straumann A, Conus S, Grzonka P, Kita H, Kephart G, Bussmann C, et al. Anti-interleukin-5 antibody treatment (mepolizumab) in active eosinophilic oesophagitis: a randomised, placebocontrolled, double-blind trial. Gut. 2010 Jan;59(1):21-30.

70. Parulekar AD, Boomer JS, Patterson BM, Yin-Declue H, Deppong CM, Wilson BS, et al. A randomized controlled trial to evaluate inhibition of T-cell costimulation in allergen-induced airway inflammation. Am J Respir Crit Care Med. 2013 Mar 1;187(5):494-501.

71. Wesemann DR, Nagler CR. The Microbiome, Timing, and Barrier Function in the Context of Allergic Disease. Immunity. 2016 Apr;44(4):728-38.

72. Cahenzli J, Köller Y, Wyss M, Geuking MB, McCoy KD. Intestinal microbial diversity during earlylife colonization shapes long-term IgE levels. Cell Host Microbe. 2013 Nov 13;14(5):559-70.

73. Hill DA, Siracusa MC, Abt MC, Kim BS, Kobuley D, Kubo M, et al. Commensal bacteria-derived signals regulate basophil hematopoiesis and allergic inflammation. Nat Med. 2012 Apr;18(4):538-46.

74. Prioult G, Nagler-Anderson C. Mucosal immunity and allergic responses: lack of regulation and/or lack of microbial stimulation? Immunol Rev. 2005 Aug;206:204-18.

75. Ai C, Zhang Q, Ding J, Wang G, Liu X, Tian F, et al. Mucosal delivery of allergen peptides expressed by Lactococcus lactis inhibit allergic responses in a BALB/c mouse model. Appl Microbiol Biotechnol. 2016 Feb;100(4):1915-24.

76. Frossard CP, Steidler L, Eigenmann PA. Oral administration of an IL-10-secreting Lactococcus lactis strain prevents food-induced IgE sensitization. J Allergy Clin Immunol. 2007 Apr;119(4):952-9.

77. Kearley J, Robinson DS, Lloyd CM. CD4+CD25+ regulatory T cells reverse established allergic airway inflammation and prevent airway remodeling. J Allergy Clin Immunol. 2008 Sep;122(3):617-24.e6.

78. Kearley J, Barker JE, Robinson DS, Lloyd CM. Resolution of airway inflammation and hyperreactivity after in vivo transfer of CD4+CD25+ regulatory T cells is interleukin 10 dependent. J Exp Med. 2005 Dec 5;202(11):1539-47.

79. Klatzmann D, Abbas AK. The promise of low-dose interleukin-2 therapy for autoimmune and inflammatory diseases. Nat Rev Immunol. 2015 Apr 17;15(5):283-94.

80. Royal RE, Steinberg SM, Krouse RS, Heywood G, White DE, Hwu P, et al. Correlates of response to IL-2 therapy in patients treated for metastatic renal cancer and melanoma. Cancer J Sci Am. 1996 Apr;2(2):91-8.

81. Nizar S, Copier J, Meyer B, Bodman-Smith M, Galustian C, Kumar D, et al. T-regulatory cell modulation: the future of cancer immunotherapy? Br J Cancer. 2009 Jun 2;100(11):1697-703.

82. Grinberg-Bleyer Y, Baeyens A, You S, Elhage R, Fourcade G, Gregoire S, et al. IL-2 reverses established type 1 diabetes in NOD mice by a local effect on pancreatic regulatory $T$ cells. J Exp Med. 2010 Aug 30;207(9):1871-8.

83. Boyman O, Surh CD, Sprent J. Potential use of IL-2/anti-IL-2 antibody immune complexes for the treatment of cancer and autoimmune disease. Expert Opin Biol Ther. 2006 Dec;6(12):1323-31. 
84. Lee S-Y, Cho M-L, Oh H-J, Ryu J-G, Park M-J, Jhun J-Y, et al. Interleukin-2/anti-interleukin-2 monoclonal antibody immune complex suppresses collagen-induced arthritis in mice by fortifying interleukin-2/STAT5 signalling pathways. Immunology. 2012 Dec;137(4):305-16.

85. Dinh TN, Kyaw TS, Kanellakis P, To K, Tipping P, Toh B-H, et al. Cytokine therapy with interleukin-2/anti-interleukin-2 monoclonal antibody complexes expands CD4+CD25+Foxp3+ regulatory $T$ cells and attenuates development and progression of atherosclerosis. Circulation. 2012 Sep 4;126(10):1256-66.

86. Liu R, Zhou Q, La Cava A, Campagnolo DI, Van Kaer L, Shi F-D. Expansion of regulatory T cells via IL-2/anti-IL-2 mAb complexes suppresses experimental myasthenia. Eur J Immunol. 2010 Jun;40(6):1577-89.

87. Hao J, Campagnolo D, Liu R, Piao W, Shi S, Hu B, et al. Interleukin-2/interleukin-2 antibody therapy induces target organ natural killer cells that inhibit central nervous system inflammation. Ann Neurol. 2011 Apr;69(4):721-34.

88. Villalta SA, Rosenthal W, Martinez L, Kaur A, Sparwasser T, Tidball JG, et al. Regulatory T cells suppress muscle inflammation and injury in muscular dystrophy. Sci Transl Med. 2014 Oct 15;6(258):258ra142.

89. Kasahara K, Sasaki N, Yamashita T, Kita T, Yodoi K, Sasaki Y, et al. CD3 antibody and IL-2 complex combination therapy inhibits atherosclerosis by augmenting a regulatory immune response. J Am Heart Assoc. 2014;3(2):e000719.

90. Brandenburg S, Takahashi T, de la Rosa M, Janke M, Karsten G, Muzzulini T, et al. IL-2 induces in vivo suppression by CD4+CD25+Foxp3+ regulatory T cells. Eur J Immunol. 2008 Jun $1 ; 38(6): 1643-53$.

91. Dietrich T, Hucko T, Schneemann C, Neumann M, Menrad A, Willuda J, et al. Local delivery of IL2 reduces atherosclerosis via expansion of regulatory T cells. Atherosclerosis. 2012 Feb;220(2):329-36.

92. D’Alessio FR, Tsushima K, Aggarwal NR, West EE, Willett MH, Britos MF, et al. CD4+CD25+Foxp3+ Tregs resolve experimental lung injury in mice and are present in humans with acute lung injury. J Clin Invest. 2009 Oct;119(10):2898-913.

93. Hartemann A, Bensimon G, Payan CA, Jacqueminet S, Bourron O, Nicolas N, et al. Low-dose interleukin 2 in patients with type 1 diabetes: a phase 1/2 randomised, double-blind, placebocontrolled trial. Lancet Diabetes Endocrinol. 2013 Dec;1(4):295-305.

94. Koreth J, Matsuoka K, Kim HT, McDonough SM, Bindra B, Alyea EP, et al. Interleukin-2 and Regulatory T Cells in Graft-versus-Host Disease. N Engl J Med. 2011 Dec 1;365(22):2055-66.

95. Von Spee-Mayer C, Siegert E, Abdirama D, Rose A, Klaus A, Alexander T, et al. Low-dose interleukin-2 selectively corrects regulatory $T$ cell defects in patients with systemic lupus erythematosus. Ann Rheum Dis. 2015 Aug 31;

96. Hordinsky M, Kaplan DH. LOw-dose interleukin 2 to reverse alopecia areata. JAMA Dermatol. 2014 Jul 1;150(7):696-7.

97. Kosmaczewska A. Low-dose interleukin-2 therapy: a driver of an imbalance between immune tolerance and autoimmunity. Int J Mol Sci. 2014;15(10):18574-92. 
98. Cook KD, Kline HC, Whitmire JK. NK cells inhibit humoral immunity by reducing the abundance of CD4+ T follicular helper cells during a chronic virus infection. J Leukoc Biol. 2015 May 18;

99. Tang $\mathrm{Q}$, Henriksen $\mathrm{KJ}$, Bi M, Finger EB, Szot G, Ye J, et al. In vitro-expanded antigen-specific regulatory T cells suppress autoimmune diabetes. J Exp Med. 2004 Jun 7;199(11):1455-65.

100. Churlaud G, Jimenez V, Ruberte J, Amadoudji Zin M, Fourcade G, Gottrand G, et al. Sustained stimulation and expansion of Tregs by IL2 control autoimmunity without impairing immune responses to infection, vaccination and cancer. Clin Immunol Orlando Fla. 2014 Apr;151(2):114-26. 